\title{
Predictors of survival among 60 patients with tissue proven primary cardiac amyloidosis
}

\author{
Hussam Suradi*, Abbas Bitar*, Merrill Benson, Mithilesh K. Das \\ Krannert Institute of Cardiology, Indiana University School of Medicine, Indianapolis, USA \\ Email: hsuradi@iupui.edu \\ Received 19 March 2013; revised 23 April 2013; accepted 1 May 2013 \\ Copyright (C) 2013 Hussam Suradi et al. This is an open access article distributed under the Creative Commons Attribution License, \\ which permits unrestricted use, distribution, and reproduction in any medium, provided the original work is properly cited.
}

\begin{abstract}
Background: Cardiovascular involvement among patients with primary systemic amyloidosis (AL) is common and predicts poor prognosis. Different parameters have been used to predict outcome. We studied the prognostic significance of clinical, ECG and echocardiographic parameters of 60 patients with tissue proven primary cardiac amyloidosis. Method and Results: Records of 60 patients with primary amyloidosis and cardiac involvement documented by endomyocardial tissue biopsy were retrospectively evaluated. Patients mean age was $57.9 \pm$ 10.2 years. $71.6 \%$ were male and $86.6 \%$ Caucasian. Patients' median survival was $12.2 \pm 4.4$ months with only $50 \%$ of patients survived for more than 1 year. $60 \%$ of patients had CHF (NYHA II-IV). CHF (NYHA II-IV), IVS, LVPW and LVEF were significant on univariate survival analysis $(p<0.05)$. On multivariate analysis only CHF $(p=0.027$, HR 3.04 [95\% CI: $1.1-8.1])$ and IVS $<1.5 \mathrm{~cm}(\mathrm{p}=0.012$, HR: 3.51 [95\% CI: 1.3 - 9.3]) were predictors of survival. Patients with CHF had a median survival of $7.58 \pm$ 1.74 months contrary to those without CHF who had a median survival of $31.2 \pm 11.41$ months. Patients with IVS $\geq 1.5 \mathrm{~cm}$ had a median survival of $7.0 \pm 1.1$ months, contrary to those with an IVS $<1.5 \mathrm{~cm}$ who had a median survival of $31.9 \pm 12.4$ months. Conclusion: In patients with primary amyloidosis and cardiac involvement, length of survival is strongly associated with CHF (NYHA II-IV) and IVS compared to other electrographic and echocardiographic parameters
\end{abstract}

Keywords: Amyloidosis; Cardiomyopathy

\section{INTRODUCTION}

Light chain or primary systemic amyloidosis (AL) is a

*Authors contributed equally to the manuscript. plasma cell dyscrasia that involves extracellular pathological deposition of insoluble fibrillar proteins in organs and tissues leading to multiple organ failure and death [1]. The age adjusted incidence of primary systemic amyloidosis is estimated to be 5.1 to 12.8 per million person-years [2]. Cardiovascular involvement is common among patients with primary systemic amyloidosis with $50 \%$ of patients experiencing some cardiac manifestations [1]. Infiltration of the heart by insoluble protein deposits in amyloidosis often results in restrictive cardiomyopathy that manifests late in its course with heart failure and conduction abnormalities [3,4]. It is estimated that more than $25 \%$ of patients with primary amyloidosis will have congestive heart failure [5].

Amyloid fibrils may also deposit in myocardial vessels and valves causing local ischemia and valvular dysfunction $[1,6]$. Cardiac amyloidosis is associated with a variable but generally poor prognosis with an estimated survival of less than 6 months in untreated patients with heart failure [5,7].

Recognition of important prognostic factors is useful to counsel patients with regard to realistic expectations for the future, both in terms of survival and supportive care that will ultimately be required. With the introduction of organ transplantation, aggressive chemotherapy and bone marrow transplantation for amyloidosis, stratification for patient risk also becomes important in selection for participation in treatment studies. Nevertheless, various parameters have been proposed for risk stratification, but failed consistently in the identification of high risk patients.

The purpose of this study is to look for useful clinical, echocardiographic and electrocardiographic features that will help to determine prognosis in patients with primary cardiac amyloidosis.

\section{METHODS}

\subsection{Study Population}

This was a retrospective observational study of 60 pat- 
ients who were referred to Indiana University Medical Institutions between January 1980 and January 2007 for management of histologically proven primary systemic amyloidosis. Cardiac amyloidosis in those patients was ascertained with an endomyocardial biopsy (EMB) in 32 patients and with autopsy in 28 patients.

Approval for this study was obtained from Indiana University Institutional Review Board. Patients were referred for evaluation of cardiac involvement in the setting of established primary AL amyloidosis. Patients with secondary amyloidosis or with localized, familial, or senile amyloidosis were excluded. Cardiac amyloidosis in those patients was ascertained either with an endomyocardial biopsy or with autopsy. A detailed examination of patients' medical records was performed to determine accurate mortality information. In case of missing or unclear data, the National Death Registry was used to confirm the status of the remainder.

A review of the medical records was performed by a team of physicians blinded to the patient outcome. The following information was reviewed and recorded: demographic data, New York Heart Association (NYHA) heart failure class, ECG findings, and echocardiographic results. Patients were followed up until death or for at least 1 year after the diagnosis of cardiac amyloidosis.

The electrocardiograms of patients who visited Indiana University hospitals were analyzed for the following characteristics: rhythm, conduction abnormalities (i.e., AV block, right or left bundle branch block), a lowvoltage pattern (defined by total height of the QRS complex in the limb leads $<5 \mathrm{~mm}$ and $<10 \mathrm{~mm}$ in the precordial leads), a pseudo-infarction pattern (pathologic Q waves on the ECG, but no coronary artery disease by angiography), and a left or right atrial abnormality. The echocardiograms were analyzed for the following characteristics: inter-ventricular septal (IVS) thickness, posterior wall thickness (LVPW), left ventricular (LV) diastolic diameter, LV systolic diameter, atrial size, pericardial effusion size (if present), left ventricular ejection fraction (LVEF), and granular/sparking appearance of the myocardium by visual inspection.

Endomyocardial biopsies were obtained in the standard fashion from patients with suspicion of cardiac amyloidosis. The biopsies were analyzed with a standard hematoxylin-eosin preparation. All biopsies were also stained with Congo red to confirm the presence of amyloidosis. If the Congo red staining was equivocal, the biopsy was reviewed by electron microscopy to confirm the diagnosis. Finally, all biopsies with amyloidosis underwent immune-histochemical staining to help ascertain the exact etiology of the amyloid.

\subsection{Statistical Analysis}

Baseline demographic and clinical variables are descrip- tively summarized. Continuous variables are expressed as mean $\pm \mathrm{SD}$. Categorical data are presented as percent frequency. Student's $t$ test was used to compare differences between 2 groups for continuous variables and chi-square test was used for categorical variables. Survival analysis was performed using univariate and multivariate Cox proportional hazards and hazards ratio of the individually tested variables were calculated. Univariate survival analysis is graphically demonstrated using Kaplan-Meier curves. Data collection and analysis was performed using STATA 12. A $p$ value $<0.05$ was considered statistically significant.

\section{RESULTS}

A total of 60 patients with histologically proven primary cardiac amyloidosis were studied. Cardiac amyloidosis in those patients was ascertained with an EMB in 32 patients and with autopsy in 28 patients. Mean age was $57.9 \pm 10.2$ years, $70 \%$ were male and $86.6 \%$ were Caucasians. $71.6 \%$ of patients had multiorgan involvement (defined as two or more organs affected by amyloidosis) with $72.3 \%$ having a positive pyrophosphate scan. Congestive heart failure (NYHA II-IV) was present in $60 \%$ of patients (Table 1).

Electrocardiographic analysis revealed a low voltage among $54.2 \%$ of patients. $57.6 \%$ of patients had an abnormal QRS axis and $51.7 \%$ of patients had a pseudo infarct pattern with no previous history of a myocardial infarct with $17.2 \%, 27.5 \%, 3.4 \%, 15.5 \%$ and $1.7 \%$ having a septal, inferior, anterior, antero-septal and an-

Table 1. Demographic of patients with primary cardiac amyloidosis.

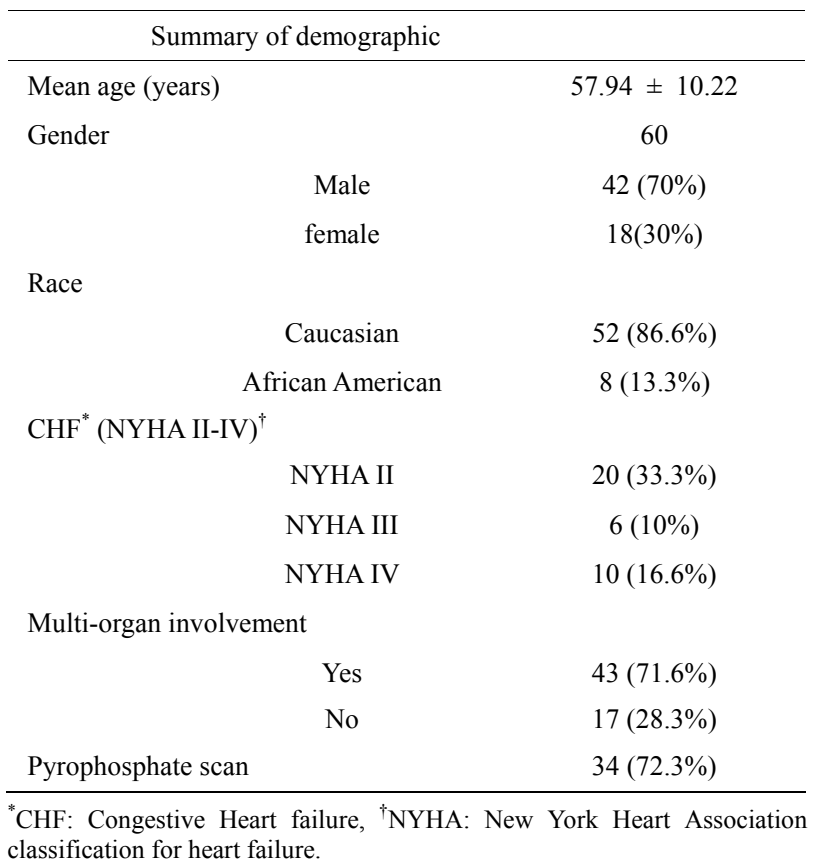


tero-lateral infarct pattern respectively. $80.3 \%$ of patients were in normal sinus rhythm with $71.6 \%$ of patients had conduction abnormalities. $21.6 \%$ of patients having a first degree block and $6.6 \%$ of patients having a second degree AV block. Right bundle branch block, left bundle branch block, intraventricular conduction delay and left anterior hemiblock were present among 13.3\%, 3.3\%, $3.3 \%$ and $30 \%$ of patients respectively. Average QTc was $448.6 \pm 37.6 \mathrm{~ms}$ with $58.9 \%$ of patients having a QTc > $440 \mathrm{~ms}$. Abnormal T wave was present among $50 \%$ of patients with $25.8 \%$ and $32.7 \%$ of patients having low flat and inverted $\mathrm{T}$ wave respectively (Table 2).

2D echocardiogram analysis revealed left ventricular hypertrophy (LVH) in $82.7 \%$ of patients. LVEF and RVSP were $0.47 \pm 0.13$ and $40.8 \pm 10.3 \mathrm{mmHg}$ respectively. The mean interventricular thickness and left posterior wall thickness were $1.46 \pm 0.34$ and $1.43 \pm 0.33$ $\mathrm{cm}$ respectively. Mitral regurgitation (MR), left atrial enlargement, speckled appearance and pericardial effusion were present among $62 \%, 63.1 \%, 8.7 \%$ and $42.1 \%$ of patients respectively (Table 3 ).

Although congestive heart failure, IVS, LVPW and EF were significant on univariate survival analysis, only New York Heart Association Class and interventricularseptal wall thickness were significant predictors of mortality during follow-up on multivariate analysis (Table 4, Figures 1 and 2).

First degree AV block, abnormal flat T wave, left and right atrial dilatation, $\mathrm{LVEF}<50 \%$, IVS $\geq 1.5 \mathrm{~cm}$, LVPW thickness $>1.1 \mathrm{~cm}$ and mitral regurgitation were statistically more common among patients with $\mathrm{CHF}$ (NYHA II-IV) $(\mathrm{p}<0.01)$.

The median survival for patients with cardiac amyloidosis was $12.2 \pm 4.4$ months, $50 \%$ of patients survived for more than 1 year. Patients with CHF (NYHA II-IV) had a median survival of $7.5 \pm 1.7$ months contrary to those without CHF who had a median survival of $31.2 \pm$ 11.4 months. Moreover, patients with IVS $\geq 1.5 \mathrm{~cm}$ had a median survival of $7.0 \pm 1.1$ months, contrary to those with an IVS $<1.5 \mathrm{~cm}$ who had a median survival of 31.9 \pm 12.4 months.

\section{DISCUSSION}

In our study we examined clinical, electrocardiographic and echocardiographic variables in relation to survival in a cohort of 60 patients with histologically-proven primary cardiac amyloidosis with the finding that only New York Heart Association class and interventricularseptal wall thickness as independent predictors of mortality.

Prognosis of patients of primary amyloidosis is poor. In three series published in 1980 's, comprising almost 500 cases of primary systemic amyloidosis (AL) from the Mayo Clinic, the median survival was 14.7 months or less [8-10]. Since then few studies assessed median sur-
Table 2. Electrocardiographic findings in patients with primary cardiac amyloidosis.

\begin{tabular}{|c|c|}
\hline \multicolumn{2}{|l|}{ Electrocardiogram Findings } \\
\hline Low voltage $\mathrm{EKG}^{*}$ & $32(54.2 \%)$ \\
\hline Pseudo-infarction & $30(51.7 \%)$ \\
\hline Yes & $28(48.2 \%)$ \\
\hline No & \\
\hline Septal & $10(17.2 \%)$ \\
\hline Inferior & $16(27.5 \%)$ \\
\hline Anterior & $2(3.4 \%)$ \\
\hline Anteroseptal & $9(15.5 \%)$ \\
\hline Anterolateral & $1(1.7 \%)$ \\
\hline \multicolumn{2}{|l|}{ Normal conduction } \\
\hline Yes & $43(71.6 \%)$ \\
\hline No & $17(28.3 \%)$ \\
\hline 1st degree $\mathrm{AV}^{\dagger}$ block & $13(21.6 \%)$ \\
\hline 2nd degree $\mathrm{AV}^{\dagger}$ block & $4(6.6 \%)$ \\
\hline 3rd degree $\mathrm{AV}^{\dagger}$ block & $0(0 \%)$ \\
\hline Right bundle branch block & $8(13.3 \%)$ \\
\hline Left bundle branch block & $2(3.3 \%)$ \\
\hline Interventricular conduction delay & $2(3.3 \%)$ \\
\hline Left anterior hemiblock & $18(30.0 \%)$ \\
\hline left posterior hemiblock & $0(0 \%)$ \\
\hline QTc & $448.6 \pm 37.6$ \\
\hline $\mathrm{QTc}>440 \mathrm{~ms}$ & $33(58.9 \%)$ \\
\hline sinus rhythm & $50(83.3 \%)$ \\
\hline sinus tachycardia & $9(15.0 \%)$ \\
\hline sinus bradycardia & $3(5.0 \%)$ \\
\hline Atrial fibrillation or flutter & $8(13.3 \%)$ \\
\hline $\mathrm{PVC}^{\ddagger}$ & $4(6.6 \%)$ \\
\hline $\mathrm{APC}^{\S}$ & $3(5.0 \%)$ \\
\hline \multicolumn{2}{|c|}{ Abnormal T wave } \\
\hline Yes & $29(50 \%)$ \\
\hline No & $29(50 \%)$ \\
\hline Low flat & $15(25.8 \%)$ \\
\hline Inferior & $14(24.1 \%)$ \\
\hline Lateral & $7(12.1 \%)$ \\
\hline Anterior & $4(6.9 \%)$ \\
\hline Inverted & $19(32.7 \%)$ \\
\hline Inferior & $7(12.1 \%)$ \\
\hline Lateral & $15(25.8 \%)$ \\
\hline Anterior & $7(12.1 \%)$ \\
\hline \multicolumn{2}{|l|}{ Abnormal QRS axis } \\
\hline Yes & $34(57.6 \%)$ \\
\hline No & $25(42.3 \%)$ \\
\hline
\end{tabular}

"EKG: Electrocardiogram, ${ }^{\dagger} \mathrm{AV}$ : Atrio-ventricular, ${ }^{\dagger}$ : PVC: Premature ventricular contraction, ${ }^{\S} \mathrm{APC}$ : Premature atrial contraction

vival and predictors of survival among patients with $\mathrm{AL}$ amyloidosis and cardiac involvement [11-15]. Furthermore, the exact incidence of cardiac amyloidosis is dif- 
Table 3. Echocardiographic findings in patients with primary cardiac amyloidosis.

\begin{tabular}{lc}
\hline Echocardiographic finding & \\
\hline LV end diastolic diameter (cm) & $4.0 \pm 0.6$ \\
LV end systolic diameter (cm) & $2.8 \pm 0.7$ \\
Left atrial size (cm) & $4 \pm 0.8$ \\
IVS tickness (cm) & $1.4 \pm 0.3$ \\
Left posterior wall thickness & $1.4 \pm 0.3$ \\
Speckled appearance of myocardium & $5(8.7 \%)$ \\
LVEF $^{\dagger}$ & $0.47 \pm 0.12$ \\
Left atrial dilatation (echo) & $36(63.1 \%)$ \\
Right atrial dilatation (echo) & $8(14.2 \%)$ \\
Left ventricular dilation (echo) & $2(3.5 \%)$ \\
Right ventricular dilatation (ech0) & $3(5.3 \%)$ \\
Left ventricular hypertrophy ( echo) & $48(82.7 \%)$ \\
RVSP & $40.8 \pm 10.3$ \\
mitral regurgitation & $31(62.0 \%)$ \\
pericardial effusion & $24(42.1 \%)$ \\
\hline
\end{tabular}

*IVS: Interventricular thickness, 'LVEF: Left ventricule ejection fraction, ${ }^{\ddagger}$ RVSP: Right ventricular systolic pressur

ficult to establish; it is under-diagnosed in the elderly and most patients are asymptomatic in early stages. Few studies assessed prognostic markers in patients with histologically proven primary cardiac amyloidosis [16]. Endomyocardial biopsy remains the gold standard diagnostic test, however cardiac amyloidosis has been more frequently diagnosed indirectly by echocardiographic evidence of amyloidosis and histological confirmation of amyloid on non-cardiac tissue [16]. Cardiac involvement affects patients' performance and limits therapeutic options. Patients can rapidly deteriorate and usually have a poor prognosis. Previous reports estimated the median survival for cardiac amyloidosis as less than one year [7] and the reported mean survival after heart failure onset is 6 months [7] which is similar to what is reported in our study.

Multiple studies assessed different variables being associated with adverse outcome such as CHF, LVPW thickness, right ventricular dilatation, low EF, LA enlargement among other variables [12-15,17].

The absence of heart failure and an IVS $<1.5 \mathrm{~cm}$ were the only factors associated with better survival in our study.

LA enlargement, abnormal flat $\mathrm{T}$ wave, left/right atrial dilatation, LVEF, LVPW and MR were more common among patients with CHF (II-IV) but failed to predict a better survival outcome on multivariate analysis.

IVS has been used as a non-invasive marker for the diagnosis of cardiac amyloidosis [18]. In a recent study conducted by Austin et al., IVS failed to predict long term mortality [11]. In another study conducted by Kristen et al. [14], IVS thickness was a predictor of outcome on univariate analysis but not on multivariate analysis.

Among the echocardiographic findings in our study which might prove to be useful in risk stratification is the magnitude of IVS thickness. Those patients with IVS thickness less than $1.5 \mathrm{~cm}$ were more likely to survive for a longer period of time. Moreover, LVPW thickness was a predictor of mortality on univariate analysis but this failed to be true on multivariate analysis. This is in contrast to what is reported in the literature where LVPW thickness was a strong predictor of clinical outcomes [13, 14].

Kristen et al. reported that left ventricular ejection fraction is a predictor of mortality on multivariate analysis [14]. In our study LVEF univariate analysis was a predictor of a better survival but failed to predict any survival benefit on multivariate analysis.

Pericardial effusion may suggest a restrictive cardiomyopathy or cardiac amyloidosis; its presence might even result in low voltage on EKG [19]. In our study, around $42 \%$ of patients had pericardial effusion, this is less than what is reported by Piper et al. where $63 \%$ of patients had pericardial effusion [19]. Absence of pericardial effusion failed to predict survival benefit.

Left atrial dilatation a surrogate marker of diastolic dysfunction was present in $63 \%$ of our patients. In contrary to what is reported in the literature, absence of left atrial dilatation failed to predict any survival benefit [15].

Despite the presence of a variety of EKG findings which might be helpful in diagnosing cardiac amyloidosis, low voltage and pseudo infarct were more common than what Murtagh [20] and less than what Dubrey [5] reported in their studies. The variety of abnormal EKG findings did not have prognostic significance in our study or in other prior studies.

There are several reasons why different electrocardiographic and echocardiographic parameters have been associated with survival in some studies but not others including our study. First, study populations have been variable, with most subjects being diagnosed with cardiac amyloidosis based on noncardiac biopsy, electrocardiographic and echocardiographic changes suggestive of cardiac involvement. It is well documented that many of those parameters like ejection fraction, diastolic dysfunction, and low voltage among others may be present in patients with amyloidosis without cardiac involvement and may affect survival. On the other hand many patients with AL amyloidosis with typical non-invasive diagnostic tests suggestive of cardiac involvement were not referred for an EMB which limit the number of patients being analyzed in many of the cardiac amyloidosis studies. Moreover the use of echocardiographic measures like ejection fraction to predict prognosis might not be very accurate because its estimation depends on operator experience, technique used and presence of other medi- 
Table 4. Univariate and multivariate predictors of survival in patients with primary cardiac amyloidosis.

\begin{tabular}{|c|c|c|c|c|}
\hline & Univariate analysis & & Multivariate analysis & \\
\hline Variable & Hazard ratio $[95 \% \mathrm{CI}]$ & P value & Hazard ratio $[95 \% \mathrm{CI}]$ & $\mathrm{p}$ value \\
\hline Sex & $0.78[0.61-1.86]$ & 0.833 & & \\
\hline Race & $0.78[0.37-1.67]$ & 0.5365 & & \\
\hline CHF $^{*}$ (NYHA II-IV) ${ }^{\dagger}$ & $2.66[1.50-4.74]$ & 0.0005 & $3.04[1.135-8.187]$ & 0.027 \\
\hline Low voltage limb leads & $1.43[0.84-2.46]$ & 0.1794 & & \\
\hline Q Wave & $1.42[0.84-2.42]$ & 0.191 & & \\
\hline Conduction abnormalities & $1.47[0.82-2.62]$ & 0.2022 & & \\
\hline Rhythm abnormalities & $0.86[0.43-1.72]$ & 0.6735 & & \\
\hline $\mathrm{T}$ wave abnormalities & $1.44[0.85-2.44]$ & 0.1727 & & \\
\hline IVS $^{\ddagger}$ & $3.68[1.64-8.26]$ & 0.0024 & $3.51[1.31-9.39]$ & 0.012 \\
\hline LVPW $^{\S}$ thickness & $3.32[1.36-8.16]$ & 0.0099 & $0.86[0.20-3.77]$ & 0.845 \\
\hline Speckled appearance & $0.68[0.27-1.73]$ & 0.3982 & & \\
\hline $\operatorname{LVEF}^{\|}(45 \%)$ & $0.47[0.24-0.94]$ & 0.0397 & & \\
\hline $\operatorname{LVEF}^{\|}(50 \%)$ & $0.39[0.20-0.78]$ & 0.0092 & $1.18[0.47-2.96]$ & 0.724 \\
\hline Left atrial dilatation & $1.47[0.85-2.57]$ & 0.1572 & & \\
\hline QTc prolongation & $1.06[0.62-1.83]$ & 0.8183 & & \\
\hline Mitral regurgitation & $1[0.56-1.80]$ & 0.9751 & & \\
\hline Pericardial effusion & $1.28[0.94-1.75]$ & 0.1304 & & \\
\hline Pyrophosphate scan & $1.25[0.66-2.40]$ & 0.488 & & \\
\hline Multiorgan involvement & $0.82[0.46-1.47]$ & 0.5186 & & \\
\hline
\end{tabular}

${ }^{*} \mathrm{CHF}$ : congestive heart failure, ${ }^{\dagger} \mathrm{NYHA}$ : New York Heart association classification, ${ }^{\dagger}$ IVS: Interventricular septum thickness, ${ }^{8}$ LVPW: Left ventricular posterior wall, "LVEF: Left ventricle ejection fraction.

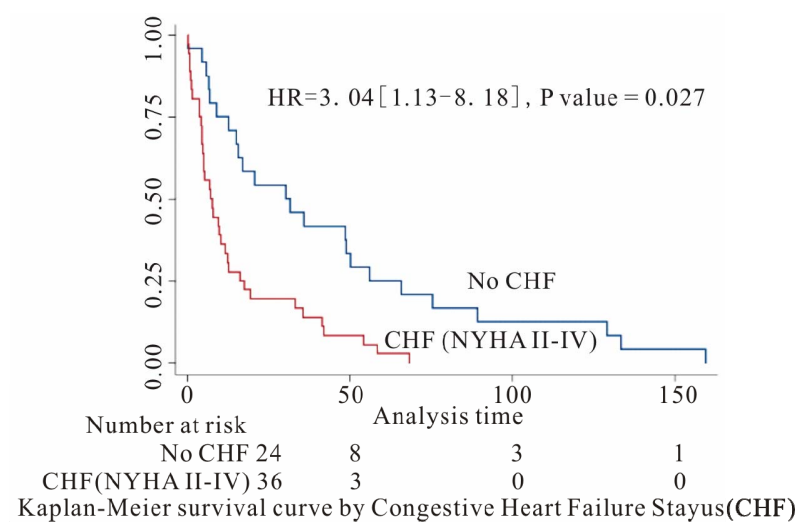

Figure 1. Kaplan-Meier survival curve by congestive heart failure (CHF) status for all-cause mortality.

cal/co-morbid conditions [21].

Among the clinical data, only the presence of $\mathrm{CHF}$ reflected poor prognosis and predicted a survival of less than one year. The presence of multi-organ involvement was not a useful predictor of survival.

Functional status, defined by NYHA class, is a strong predictor of outcome in patients with depressed or preserved LV function [22,23]. This applies to patients with cardiac amyloidosis. Our study, similar to other studies, has shown that NYHA is a strong predictor of

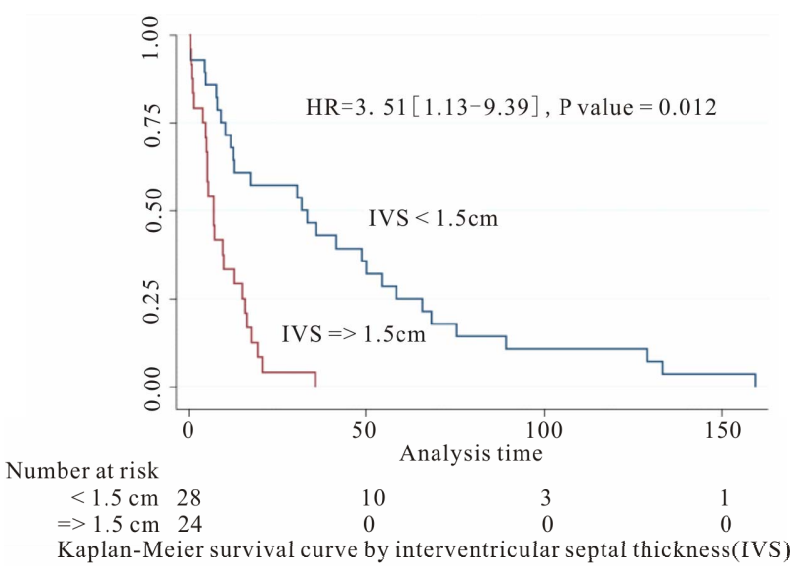

Figure 2. Kaplan-Meier survival curve by interventricularseptal thickness (IVS) for all-cause mortality.

mortality [11].

In our study the median survival for patients with cardiac amyloidosis was $12.23 \pm 4.43$ months, $50 \%$ of patients survived for more than 1 year. Our results are in accordance to what is published in the literature $[5,11$, 14,24]. Our group of patients with heart failure did better than what Kyle et al. reported [7]. This discrepancy in survival might be due to the advances in amyloidosis and heart failure therapy. 
Our study was able to identify one clinical and one echocardiographic parameter that can be used to identify patients at high risk for disease progression. Identifications of such markers early before clinical deterioration might help in prompt institution of therapy whether it is chemotherapy, bone marrow and heart transplantation [25].

The strength of our study lies in the fact that it involved 60 patients with histologically proven cardiac amyloidosis.

A limitation of this study is that it is a retrospective, single centered cohort study that included 60 patients over a long period of time. This might be due to the fact that amyloidosis is a rare disease and cardiac involvement with cardiac tissue documentation is even rarer. Moreover, other variables like troponin I/T, proBNP, cardiac MRI, E/A ratios and strain in addition to others, have been associated with mortality were missing and were not included in our study.

\section{CONCLUSIONS}

Our study indicates that patients with cardiac amyloidosis have similar outcome to what is reported in the literature. Long term survival is strongly associated with CHF (NYHA II-IV) and septal thickness compared to other electrographic and echocardiographic parameters.

Moreover, our study indicates that NYHA and IVS may be used to detect patients at high risk of mortality and help early initiation of aggressive management and treatment.

\section{REFERENCES}

[1] Falk, R.H. (2005) Diagnosis and management of the cardiac amyloidoses. Circulation, 112, 2047-2060. doi:10.1161/CIRCULATIONAHA.104.489187

[2] Falk, R.H., Comenzo, R.L. and Skinner, M. (1997) The systemic amyloidoses. The New England Journal of Medicine, 337, 898-909. doi:10.1056/NEJM199709253371306

[3] Kyle, R.A. (1995) Amyloidosis. Circulation, 91, 12691271. doi:10.1161/01.CIR.91.4.1269

[4] Chew, C., Ziady, G.M., Raphael, M.J. and Oakley, C.M. (1975) The functional defect in amyloid heart disease. The "stiff heart" syndrome. American Journal of Cardiology, 36, 438-444. doi:10.1016/0002-9149(75)90891-7

[5] Dubrey, S.W., Cha, K., Anderson, J., Chamarthi, B., Reisinger, J., Skinner, M. and Falk, R. H. (1998) The clinical features of immunoglobulin light-chain (AL) amyloidosis with heart involvement. Qurom Journal Manager, 91, 141-157. doi:10.1093/qjmed/91.2.141

[6] Mueller, P.S., Edwards, W.D. and Gertz, M.A. (2000) Symptomatic ischemic heart disease resulting from obstructive intramural coronary amyloidosis. American Journal of Medicine, 109, 181-188.

\section{doi:10.1016/S0002-9343(00)00471-X}

[7] Kyle, R.A. and Gertz, M.A. (1995) Primary systemic amyloidosis: Clinical and laboratory features in 474 cases. Seminars in Hematology, 32, 45-59.

[8] Kyle, R.A. and Greipp, P.R. (1983) Amyloidosis (AL). Clinical and laboratory features in 229 cases. Mayo Clinic Proceedings, 58, 665-683.

[9] Kyle, R.A. and Bayrd, E.D. (1975) Amyloidosis: Review of 236 cases. Medicine (Baltimore), 54, 271-299. doi:10.1097/00005792-197507000-00001

[10] Kyle, R.A. and Bayrd, E.D. (1961) Primary systemic amyloidosis and myeloma. Discussion of relationship and review of 81 cases. Archives of Internal Medicine, 107, 344-353. doi:10.1001/archinte.1961.03620030032004

[11] Austin, B.A., Duffy, B., Tan, C., Rodriguez, E.R., Starling, R.C. and Desai, M.Y. (2009) Comparison of functional status, electrocardiographic, and echocardiographic parameters to mortality in endomyocardial-bopsy proven cardiac amyloidosis. American Journal of Cardiology, 103, 1429-1433. doi:10.1016/j.amjcard.2009.01.361

[12] Cueto-Garcia, L., Reeder, G.S., Kyle, R.A., Wood, D.L., Seward, J.B., Naessens, J., Offord, K.P., Greipp, P.R., Edwards, W.D. and Tajik, A.J. (1985) Echocardiographic findings in systemic amyloidosis: Spectrum of cardiac involvement and relation to survival. Journal of the American College of Cardiology, 6, 737-743. doi:10.1016/S0735-1097(85)80475-7

[13] Koyama, J., Ray-Sequin, P.A. and Falk, R.H. (2002) Prognostic significance of ultrasound myocardial tissue characterization in patients with cardiac amyloidosis. Circulation, 106, 556-561. doi:10.1161/01.CIR.0000023530.86718.B0

[14] Kristen, A.V., Perz, J.B., Schonland, S.O., Hegenbart, U., Schnabel, P.A., Kristen, J.H., Goldschmidt, H., Katus, H. A. and Dengler, T.J. (2007) Non-invasive predictors of survival in cardiac amyloidosis. European Journal of Heart Failure, 9, 617-624. doi:10.1016/j.ejheart.2007.01.012

[15] Mohty, D., Pibarot, P., Dumesnil, J.G., Darodes, N., Lavergne, D., Echahidi, N., Virot, P., Bordessoule, D. and Jaccard, A. (2011) Left atrial size is an independent predictor of overall survival in patients with primary systemic amyloidosis. Archives of Cardiovascular Diseases, 104, 611-618. doi:10.1016/j.acvd.2011.10.004

[16] Kapoor, P., Thenappan, T., Singh, E., Kumar, S. and Greipp, P.R. (2011) Cardiac amyloidosis: A practical approach to diagnosis and management. American Journal of Medicine, 124, 1006-1015. doi:10.1016/j.amjmed.2011.04.013

[17] Patel, A.R., Dubrey, S.W., Mendes, L.A., Skinner, M., Cupples, A., Falk, R.H. and Davidoff, R. (1997) Right ventricular dilation in primary amyloidosis: An independent predictor of survival. American Journal of Cardiology, 80, 486-492. doi:10.1016/S0002-9149(97)00400-1

[18] Rahman, J.E., Helou, E.F., Gelzer-Bell, R., Thompson, R. E., Kuo, C., Rodriguez, E.R., Hare, J.M., Baughman, K. L. and Kasper, E.K. (2004) Noninvasive diagnosis of biopsy-proven cardiac amyloidosis. Journal of the Ameri- 
can College of Cardiology, 43, 410-415. doi:10.1016/j.jacc.2003.08.043

[19] Piper, C., Butz, T., Farr, M., Faber, L., Oldenburg, O. and Horstkotte, D. (2010) How to diagnose cardiac amyloidosis early: Impact of ECG, tissue Doppler echocardiography, and myocardial biopsy. Amyloid, 17, 1-9. doi:10.3109/13506121003619310

[20] Murtagh, B., Hammill, S.C., Gertz, M.A., Kyle, R.A., Tajik, A.J. and Grogan, M. (2005) Electrocardiographic findings in primary systemic amyloidosis and biopsyproven cardiac involvement. American Journal of Cardiology, 95, 535-537. doi:10.1016/j.amjcard.2004.10.028

[21] Bellenger, N.G., Burgess, M.I., Ray, S.G., Lahiri, A., Coats, A.J., Cleland, J.G. and Pennell, D.J. (2000) Comparison of left ventricular ejection fraction and volumes in heart failure by echocardiography, radionuclide ventriculography and cardiovascular magnetic resonance; are they interchangeable? European Heart Journal, 21, 13871396. doi:10.1053/euhj.2000.2011
[22] Jones, R.C., Francis, G.S. and Lauer, M.S. (2004) Predictors of mortality in patients with heart failure and preserved systolic function in the Digitalis Investigation Group trial. Journal of the American College of Cardiology, 44, 1025-1029. doi:10.1016/j.jacc.2004.05.077

[23] Pocock, S.J., Wang, D., Pfeffer, M.A., Yusuf, S., McMurray, J.J., Swedberg, K.B., Ostergren, J., Michelson, E.L., Pieper, K.S. and Granger, C.B. (2006) Predictors of mortality and morbidity in patients with chronic heart failure. European Heart Journal, 27, 65-75. doi:10.1093/eurheartj/ehi555

[24] Carroll, J.D., Gaasch, W.H. and McAdam, K.P. (1982) Amyloid cardiomyopathy: Characterization by a distinctive voltage/mass relation. American Journal of Cardiology, 49, 9-13. doi:10.1016/0002-9149(82)90270-3

[25] Selvanayagam, J.B., Hawkins, P.N., Paul, B., Myerson, S. G. and Neubauer, S. (2007) Evaluation and management of the cardiac amyloidosis. American Journal of Cardiology, 50, 2101-2110. doi:10.1016/j.jacc.2007.08.028 